\title{
THE NUMERICAL CALCULATION OF TRAVELING WAVE SOLUTIONS OF NONLINEAR PARABOLIC EQUATIONS*
}

\author{
THOMAS HAGSTROM $\dagger$ AND H. B. KELLER $\ddagger$
}

\begin{abstract}
Traveling wave solutions have been studied for a variety of nonlinear parabolic problems. In the initial value approach to such problems the initial data at infinity determines the wave that propagates. The numerical simulation of such problems is thus quite difficult. If the domain is replaced by a finite one, to facilitate numerical computations, then appropriate boundary conditions on the "artificial" boundaries must depend upon the initial data in the discarded region. In this work we derive such boundary conditions, based on the Laplace transform of the linearized problems at $\pm \infty$, and illustrate their utility by presenting a numerical solution of Fisher's equation which has been proposed as a model in genetics.
\end{abstract}

Key words. artificial boundary conditions, parabolic traveling waves

AMS(MOS) subject classifications. 65M99, 35K15

1. Introduction. We develop numerical methods for computing traveling wave solutions of Cauchy problems for nonlinear parabolic equations posed on infinite spatial domains. In particular we consider the general one space dimensional case:
a) $u_{t}=f\left(u_{x x}, u_{x}, u\right), \quad-\infty<x<\infty, \quad t>0$,
b) $\lim _{x \rightarrow \pm \infty} u(x, t)=\varphi_{ \pm}$,
c) $u(x, 0)=u_{0}(x)$.

The equation (1.1a) is parabolic if, as we assume:

$$
\text { d) } \frac{\partial}{\partial a} f(a, b, c) \geqq 1 \quad \forall a, b, c \in \mathbb{R} .
$$

Further we require that the constant states at $\pm \infty$ are compatible with smooth steady states, but that changes in their values effect the wave; thus:
a) $f\left(0,0, \varphi_{ \pm}\right)=0$,
b) $f_{u}\left(0,0, \varphi_{ \pm}\right) \neq 0$.

Finally the initial data is required to satisfy $(1.1 \mathrm{~b})$; that is:

c) $\lim _{x \rightarrow \pm \infty} u_{0}(x)=\varphi_{ \pm}$

In particular we wish to compute the evolution of initial data into traveling waves, that is into solutions of the form $u=w(x-c t)$.

Hagan [3], [4] has presented an extensive analysis of problem (1.1). We paraphrase some of his results below:

(i) Nonmonotonic waves (i.e. $w^{\prime}(\xi)$ not of one sign) are unstable in general.

\footnotetext{
* Received by the editors May 24, 1984, and in revised form July 1, 1985. This research was sponsored in part by the U.S. Department of Energy under contract DE-AS03-76SF-00767, and by the U.S. Army under contract DAAG29-80-C-0041.

$\dagger$ Department of Applied Mathematics and Statistics, State University of New York at Stony Brook, Stony Brook, New York 11794.

¥Department of Applied Mathematics, California Institute of Technology, Pasadena, California 91125.
} 
(ii) The stability of monotonic waves of speed $c$ can, in general, be determined by an examination of their trajectories in the phase plane of:

$$
w^{\prime}=v, \quad f\left(v^{\prime}, v, w\right)+c v=0 .
$$

(iii) If traveling waves exist, a large class of initial data, $u_{0}(x)$, satisfying $(1.1 \mathrm{~b})$ will evolve to the same traveling wave.

(iv) Infinitely many wavespeeds, $c$, may be allowed. In this case, the traveling wave which evolves depends on the behavior of the initial data at infinity.

The numerical solution of (1.1) requires a finite computational domain. One way to obtain such a domain is to introduce artificial boundaries at the points $x=\tau_{ \pm}$, $\tau_{+}>\tau_{-}$. The derivation of such boundary conditions is the main subject of this work. A general theory of boundary conditions at an artificial boundary is given by the authors in [6]. This theory is not directly applicable to time dependent problems in unbounded spatial domains such as (1.1). However, a Laplace transformation in time yields a problem of the right form. In $\S 2$, proper boundary conditions are derived for the transformed problem and they are inverted to yield conditions on the direct problem.

We note that use of the proper boundary conditions is crucial whenever (iv) holds. Then, the "naive" conditions, replacing (1.1b) by:

$$
u\left(\tau_{ \pm}, t\right)=\varphi_{ \pm}
$$

must, in general, fail to lead to the correct long time solution.

In $\S 3$ a specific problem of the form (1.1) is introduced: the Cauchy problem for Fisher's equation. It has traveling wave solutions of all speeds $c \geqq 2$. Gazdag and Canosa [1] present a numerical solution of Fisher's equation using boundary conditions analogous to (1.4). As predicted by the theory, their solution always evolved to the traveling wave of minimum speed, $c=2$. In order to calculate traveling waves with speeds $c \geqq 2$, we employ the boundary conditions derived in $\S 2$. The numerical solution evolves to the correct traveling wave for a variety of choices of initial data.

We note that the method of deriving boundary conditions presented here can be applied to other time dependent problems, including some problems of hyperbolic type. For other examples the reader is referred to Gustafsson and Kreiss [2] and Hagstrom [5].

2. Construction of the boundary conditions. We construct boundary conditions at the right-hand boundary, $x=\tau_{+}$. The construction at the left will be analogous. Although the general results of [6] are not directly applicable to this problem, they do motivate our approach and, hence, we summarize them below.

For a linear problem the exact boundary condition at the artificial boundary is characterized in the following way: the Cauchy data at the artificial boundary, that is the function along with the correct number of its normal derivatives, should be an element of a certain affine set. This affine set is determined by the subspace of Cauchy data which leads to solutions of the homogeneous problem in the tail, $x \geqq \tau_{+}$, combined with the trace of any particular solution of the inhomogeneous problem. For certain nonlinear problems whose solution is required to have a smooth limit at infinity, the existence of an exact nonlinear boundary condition is established. Here it is necessary to assume that the solution at the artificial boundary is sufficiently close to its limiting value. An asymptotic expansion of the boundary condition is derived, the first term of which consists of the boundary condition for the problem linearized about the limiting solution.

Given this last fact about nonlinear problems, it is reasonable to analyze the problem in the tail linearized about $\varphi_{+}$. We assume a coordinate system moving to the 
right with speed $c$ and thus we consider:
a) $v(x, t)=u(x, t)-\varphi_{+}, \quad x \geqq \tau_{+}$,
b) $v_{t}=f_{1} v_{x x}+f_{2} v_{x}+c v_{x}+f_{3} v$
c) $v(x, 0)=u_{0}(x)-\varphi_{+}$,
d) $\lim _{x \rightarrow \infty} v(x, t)=0$.

Here the constants $f_{i}$ are given by:

$$
f_{1}=\frac{\partial f}{\partial\left(u_{x x}\right)}\left(0,0, \varphi_{+}\right), \quad f_{2}=\frac{\partial f}{\partial\left(u_{x}\right)}\left(0,0, \varphi_{+}\right), f_{3}=\frac{\partial f}{\partial u}\left(0,0, \varphi_{+}\right)
$$

Following the discussion above, to derive boundary conditions from (2.1) two problems must be solved; boundary conditions for the homogeneous problem, $(2.1 \mathrm{~b}$, d) combined with zero initial data, must be found as well as a particular solution of $(2.1 \mathrm{~b}, \mathrm{~d})$ which satisfies $(2.1 \mathrm{c})$. The homogeneous problem is considered first.

2.1. Boundary conditions for the homogeneous problem. We introduce the temporal Laplace transform:

$$
\hat{w}(x, s)=\int_{0}^{\infty} e^{-s t} w(x, t) d t
$$

If $w$ is a solution of $(2.1 \mathrm{~b}, \mathrm{~d})$ with zero initial data, then $\hat{w}$ satisfies:
a) $f_{1} \hat{w}_{x x}+\left(f_{2}+c\right) \hat{w}_{x}+\left(f_{3}-s\right) \hat{w}=0$,
b) $\lim _{x \rightarrow \infty} \hat{w}(x, s)=0$.

Equation (2.3a) has the basic exponential solutions:

$$
\hat{w}=e^{\kappa_{ \pm}(s ; c) x},
$$

where $\kappa_{ \pm}(s ; c)$ are given by:

$$
\kappa_{ \pm}=\frac{-\left(f_{2}+c\right)}{2 f_{1}} \pm \frac{1}{2 f_{1}}\left[\left(f_{2}+c\right)^{2}+4 f_{1}\left(s-f_{3}\right)\right]^{1 / 2} .
$$

For $\operatorname{Re}(s)$ sufficiently large, $\operatorname{Re}\left(\kappa_{+}\right) \geqq 0$ and $\operatorname{Re}\left(\kappa_{-}\right) \leqq 0$, since $f_{1} \geqq 1$. Hence, the admissible solution has exponent $\kappa_{-}$and it satisfies:

$$
\hat{w}_{x}\left(\tau_{+} ; s\right)=\kappa_{-}(s ; c) \hat{w}\left(\tau_{+} ; s\right) .
$$

This can be rewritten as:

$$
\frac{\hat{w}_{x}\left(\tau_{+} ; s\right)}{\kappa_{-}(s ; c)}=\hat{w}\left(\tau_{+} ; s\right) .
$$

Using the convolution formulas and the expression for the inverse transform of $1 / \kappa$ (see, e.g., Oberhettinger and Badii [9]), (2.7) yields for $w\left(\tau_{+}, t\right)$ the condition:

$$
-\sqrt{f_{1}} \int_{0}^{t}\left\{e^{\left[f_{3}-\alpha^{2}\right](t-p)}\left[\frac{1}{\sqrt{\pi(t-p)}}-\alpha e^{\alpha^{2}(t-p)} \operatorname{Erfc}(\alpha \sqrt{t-p})\right] w_{x}\left(\tau_{+}, p\right)\right\} d p=w\left(\tau_{+}, t\right)
$$


where we have introduced:

$$
\alpha \equiv \frac{f_{2}+c}{2 \sqrt{f_{1}}}
$$

2.2. Particular solution. We now find particular solutions in the case that $u_{0}(x)-$ $\varphi_{+}$is expressed as a finite sum of exponentials:

$$
u_{0}(x)-\varphi_{+} \equiv \sum_{j=1}^{N} d_{j} e^{-\alpha_{j}\left(x-\tau_{+}\right)}, \quad \alpha_{j}>0, \quad x \geqq \tau_{+} .
$$

Hagan's analysis [3] shows that $u_{0}(x)-\varphi_{+}$must decay at least exponentially for traveling wave solutions to exist. From (2.5), with $s=0$, we see that traveling waves of speed $c$ have exponential decay rates given by:

$$
g_{ \pm}(c)=-\frac{f_{2}+c}{2 f_{1}} \pm \frac{1}{2 f_{1}}\left[\left(f_{2}+c\right)^{2}-4 f_{1} f_{3}\right]^{1 / 2}
$$

Thus for any exponent, $-\alpha_{j}$, there exists a unique speed $c_{j}$ such that:

$$
-\alpha_{j}=g_{-}\left(c_{j}\right) \text { or }-\alpha_{j}=g_{+}\left(c_{j}\right) \text {. }
$$

This speed is given by:

$$
c_{j}=\frac{f_{3}}{\alpha_{j}}+f_{1} \alpha_{j}-f_{2} .
$$

Hence, each exponential in (2.9) can be associated with a unique traveling wave, from which a particular solution can be found. This gives the particular solution corresponding to the initial data (2.9):

$$
v_{p}(x, t)=\sum_{j=1}^{N} d_{j} e^{-\alpha_{j}\left(\left[x-\tau_{+}\right]-\left(c-c_{j}\right) t\right)} .
$$

Combining (2.8) and (2.12) yields the following linearized boundary condition on $u \equiv w-v_{p}$ at $x=\tau_{+}$:

$$
\begin{aligned}
& -\sqrt{f_{1}} \int_{0}^{t}\left\{e^{\left[f_{3}-\alpha^{2}\right](t-p)}\left[\frac{1}{\sqrt{\pi(t-p)}}-\alpha e^{\alpha^{2}(t-p)} \operatorname{Erfc}(\alpha \sqrt{t-p})\right]\right. \\
& \left.\cdot\left(u_{x}\left(\tau_{+}, p\right)+\sum_{j=1}^{N} \alpha_{j} d_{j} e^{\alpha_{j}\left(c_{j}-c\right) p}\right)\right\} d p \\
& =u\left(\tau_{+}, t\right)-\varphi_{+}-\sum_{j=1}^{N} d_{j} e^{\alpha_{j}\left(c_{j}-c\right) t} .
\end{aligned}
$$

Here of course:
a) $\alpha=\frac{f_{2}+c}{2 \sqrt{f_{1}}}$
b) $u_{0}(x)=\varphi_{+}+\sum_{j=1}^{N} d_{j} e^{-\alpha_{j}\left(x-\tau_{+}\right)}, \quad x \geqq \tau_{+}$.

2.3. Conditions at the left boundary. A similar boundary condition can be derived at the left-hand boundary, $x=\tau_{-}$. In transform variables, a solution to the linearized, 
homogeneous problem on $\left(-\infty, \tau_{-}\right]$must satisfy:

$$
\frac{\hat{w}_{x}\left(\tau_{-} ; s\right)}{\bar{\kappa}_{+}(s ; c)}=\hat{w}\left(\tau_{-} ; s\right),
$$

where

$$
\bar{\kappa}_{+}=\frac{-\left(\bar{f}_{2}+c\right)}{2 \bar{f}_{1}}+\frac{1}{2 \bar{f}_{1}}\left[\left(\bar{f}_{2}+c\right)^{2}+4 \bar{f}_{1}\left(s-\bar{f}_{3}\right)\right]^{1 / 2}
$$

and

$$
\bar{f}_{1}=\frac{\partial f}{\partial\left(u_{x x}\right)}\left(0,0, \varphi_{-}\right), \quad \bar{f}_{2}=\frac{\partial f}{\partial\left(u_{x}\right)}\left(0,0, \varphi_{-}\right), \quad \bar{f}_{3}=\frac{\partial f}{\partial u}\left(0,0, \varphi_{-}\right)
$$

The inverse transform of (2.15) is given by:

$$
\sqrt{\bar{f}_{1}} \int_{0}^{t}\left\{e^{\left[\bar{f}_{3}-\bar{\alpha}^{2}\right](t-p)}\left[\frac{1}{\sqrt{\pi(t-p)}}+\bar{\alpha} e^{\bar{\alpha}^{2}(t-p)} \operatorname{Erfc}(-\bar{\alpha} \sqrt{t-p})\right] w_{x}\left(\tau_{-}, p\right)\right\} d p=w\left(\tau_{-}, t\right)
$$

with $\bar{\alpha}=\left(\bar{f}_{2}+c\right) / 1 \sqrt{f_{1}}$. To find a particular solution, we assume that

$$
u_{0}(x)-\varphi_{-}=\sum_{j=1}^{M} \bar{d}_{j} e^{\bar{\alpha}_{j}\left(x-\tau_{-}\right)}, \quad \bar{\alpha}_{j}>0, \quad x \leqq \tau_{-} .
$$

Each exponent, $\bar{\alpha}_{j}$, can be uniquely associated with a linear traveling wave of speed $\bar{c}_{j}$ through (2.16), with $s=0$ :

$$
\bar{c}_{j}=-\bar{f}_{2}-\bar{f}_{1} \bar{\alpha}_{j}-\frac{\bar{f}_{3}}{\bar{\alpha}_{j}} .
$$

This leads to the particular solution:

$$
\bar{V}_{p}(x, t)=\varphi_{-}+\sum_{j=1}^{M} \bar{d}_{j} e^{-\bar{\alpha}_{j} \tau_{-}} e^{\bar{\alpha}_{j}\left(x+\left(c-\bar{c}_{j}\right) t\right)} .
$$

Combining (2.20) with (2.18) yields a linear boundary condition at $\tau_{-}$, analogous to (2.13):

$$
\begin{aligned}
& \sqrt{\bar{f}_{1}} \int_{0}^{t} e^{\left[\bar{f}_{3}-\bar{\alpha}^{2}\right](t-p)}\left[\frac{1}{\sqrt{\pi(t-p)}}+\bar{\alpha} e^{\bar{\alpha}^{2}(t-p)} \operatorname{Erfc}(-\bar{\alpha} \sqrt{t-p})\right] \\
& \cdot\left(u_{x}\left(\tau_{-}, p\right)-\sum_{j=1}^{M} \bar{\alpha}_{j} \bar{d}_{j} e^{\bar{\alpha}_{j}\left(c-\bar{c}_{j}\right) p}\right) d p \\
& =u\left(\tau_{-}, t\right)-\varphi_{-}-\sum_{j=1}^{M} \bar{d}_{j} e^{\bar{\alpha}_{j}\left(c-c_{j}\right) t} .
\end{aligned}
$$

Here
a) $\bar{\alpha}=\frac{\bar{f}_{2}+c}{2 \sqrt{\bar{f}_{1}}}$
b) $u_{0}(x)=\varphi_{-}+\sum_{j=1}^{M} \bar{d}_{j} e^{\bar{\alpha}_{j}\left(x-\tau_{-}\right)}, \quad x \leqq \tau_{-}$. 
3. Application to Fisher's equation. We now apply the results of the preceding section to Fisher's equation:

a) $u_{t}=u_{x x}+u(1-u), \quad x \in(-\infty, \infty), \quad t \geqq 0$,

b) $\lim _{x \rightarrow \infty} u(x, t)=0, \quad \lim _{x \rightarrow-\infty} u(x, t)=1$,

c) $u(x, 0)=u_{0}(x)$

Problem (3.1) is used as a model of the propagation of an advantageous gene. For a discussion of this application see, for example, Moran [8]. It is a special case of (1.1) and various statements concerning the behavior of its solution are consequences of Hagan's [3] general analysis. (They were first proved by Larson [7].)

(i) There exist traveling wave solutions of all wavespeeds $c \geqq 2$.

(ii) All positive initial data, $u_{0}(x)$, decaying at least exponentially as $x \rightarrow \infty$ evolves to a unique traveling wave.

(iii) If $u_{0}(x) \sim e^{-\beta x}$ as $x \rightarrow \infty$, then the solution evolves to a wave of speed $c(\beta)$ given by:

$$
c(\beta)= \begin{cases}\frac{1+\beta^{2}}{\beta}, & \beta \leqq 1, \\ 2, & \beta \geqq 1 .\end{cases}
$$

The linearized boundary conditions, (2.13) and (2.21), are easily specialized to this problem. As in $\S 2$, we introduce a coordinate system moving to the right with speed $c$ and choose $\tau_{+}$and $\tau_{-}$as our artificial boundary locations. We assume that $u_{0}(x)$ can be represented as a finite sum of exponentials in the tails:

$$
\begin{array}{ll}
u_{0}(x)=\sum_{j=1}^{N} d_{j} e^{-\alpha_{j}\left(x-\tau_{+}\right)}, & x \geqq \tau_{+}, \\
u_{0}(x)=\sum_{j=1}^{M} \bar{d}_{j} e^{\bar{\alpha}_{j}\left(x-\tau_{-}\right)}+1, & x \leqq \tau_{-} .
\end{array}
$$

The boundary conditions we impose are:

$$
\begin{aligned}
& \text { a) }-\int_{0}^{t} e^{\left(1-c^{2} / 4\right)(t-p)}\left[\frac{1}{\sqrt{\pi(t-p)}}-\frac{c}{2} e^{c^{2}(t-p) / 4} \operatorname{Erfc}\left(\frac{c}{2} \sqrt{t-p}\right)\right] \\
& \cdot\left[u_{x}\left(\tau_{+}, p\right)+\sum_{j=1}^{N} \alpha_{j} d_{j} e^{\left(1+\alpha_{j}^{2}-\alpha_{j} c\right) p}\right] d p \\
& =u\left(\tau_{+}, t\right)-\sum_{j=1}^{N} d_{j} e^{\left(1+\alpha_{j}^{2}-\alpha_{j} c\right) t}, \\
& \text { b) } \int_{0}^{t} e^{-\left(1+c^{2} / 4\right)(t-p)}\left[\frac{1}{\sqrt{\pi(t-p)}}+\frac{c}{2} e^{c^{2}(t-p) / 4} \operatorname{Erfc}\left(-\frac{c}{2} \sqrt{t-p}\right)\right] \\
& \cdot\left[u_{x}\left(\tau_{-}, p\right)-\sum_{j=1}^{M} \bar{\alpha}_{j} \bar{d}_{j} e^{\left(\bar{\alpha}_{j}^{2}+\bar{\alpha}_{j} c-1\right) p}\right] d p \\
& =u\left(\tau_{-}, t\right)-1-\sum_{j=1}^{M} \bar{d}_{j} e^{\left(\bar{\alpha}_{j}^{2}+\bar{\alpha}_{j} c-1\right) t} .
\end{aligned}
$$

We note from (3.3) that the true solution should evolve to a wave of speed $c(\beta)$ given 
by (3.2) where

$$
\beta=\min _{j}\left\{\alpha_{j}\right\}
$$

In certain circumstances the particular terms in (3.4a) have a large exponential growth in time. As this could be a source of error in a numerical computation, the integrals involving them were done exactly. This allows us to rewrite the right boundary condition:

$$
\begin{aligned}
& -\int_{0}^{t} e^{\left(1-c^{2} / 4\right)(t-p)}\left[\frac{1}{\sqrt{\pi(t-p)}}-\frac{c}{2} e^{c^{2}(t-p) / 4} \operatorname{Erfc}\left(\frac{c}{2} \sqrt{t-p}\right)\right] u_{x}\left(\tau_{+}, p\right) d p \\
& \quad=u\left(\tau_{+}, t\right)+\sum_{j=1}^{N} f_{j}(t)
\end{aligned}
$$

Here

$$
\begin{aligned}
& f_{j}(t)=\alpha_{j} d_{j}\left[\frac{-c}{2\left(1-k_{j}\right)} e^{t} \operatorname{Erfc}\left(\frac{c}{2} \sqrt{t}\right)\right. \\
& \left.+\frac{\left[k_{j}+c^{2} / 4-1\right]^{1 / 2}}{\left(1-k_{j}\right)} e^{k_{j} t} \operatorname{Erfc}\left(\sqrt{\left(k_{j}+\frac{c^{2}}{4}-1\right) t}\right)+h_{j}(t)\right] \\
& h_{j}(t)= \begin{cases}0, & \alpha_{j}=\frac{c}{2}+\sqrt{\frac{c^{2}}{4}+k_{j}-1}, \\
-2 \frac{\sqrt{c^{2} / 4+k_{j}-1}}{\left(1-k_{j}\right)} e^{k_{j} t}, & \alpha_{j}=\frac{c}{2}-\sqrt{\frac{c^{2}}{4}+k_{j}-1},\end{cases}
\end{aligned}
$$

and

$$
k_{j}=1+\alpha_{j}^{2}-\alpha_{j} c .
$$

We note that $\left(3.4 a^{\prime}\right)$ explicitly contains the different evolution of initial data with large and small decay rates.

Presented below are the results of some numerical computations of solutions of (3.1) using the boundary conditions $\left(3.4 \mathrm{a}^{\prime}, \mathrm{b}\right)$. A uniform grid was introduced and spatial derivatives were replaced by centered finite differences. The method was implicit in time and stable for the ratio of the time step to the grid size sufficiently small. With $h$ denoting the spatial mesh width and $k$ the time step we used:

$$
\begin{aligned}
& u(x, t+k)-k D_{+} D_{-} u(x, t+k)-k u(x, t+k)(1-u(x, t+k)) \\
& \quad=u(x, t-k)+k D_{+} D_{-} u(x, t-k)+u(x, t-k)(1-u(x, t-k))+2 c k D_{0} u(x, t) .
\end{aligned}
$$

Here $D_{+} D_{-}$and $D_{0}$ are standard spatial difference operators:

$$
D_{+} D_{-} f(x)=\frac{(f(x+h)-2 f(x)+f(x-h))}{h^{2}}, \quad D_{0} f(x)=\frac{(f(x+h)-f(x-h))}{2 h} \text {. }
$$

At each step the nonlinear system of difference equations was solved using Newton's method with an explicit step taken to generate the initial guess.

The boundaries were located midway between gridpoints and the integrals there were approximated by the trapezoid rule (away from the singularity). So, for example, 
at the right-hand boundary we used:

$$
\begin{aligned}
-\left[\frac{k}{2}(\right. & \left.K(t)+K_{s}(t)\right) w(0)+k \sum_{j=1}^{(t / k-2)}\left(K(t-j k)+K_{s}(t-j k)\right) w(j k) \\
+ & k K(k) w(t-k)+\frac{k}{2} K(0) w(t)+\frac{k}{2} K_{s}(k) w(t-k) \\
& \left.+\int_{t-k}^{t}\left\{(t-p) w(t-k) e^{\left(1-c^{2} / 4\right)}-(t-k-p) w(t)\right\} \frac{d p}{\sqrt{\pi(t-p)}}\right] \\
= & \frac{1}{2}\left\{u\left(\tau_{+}+\frac{h}{2}, t\right)+u\left(\tau_{+}-\frac{h}{2}, t\right)\right\}+\sum_{j=1}^{N} f_{j}(t) .
\end{aligned}
$$

Here, $K$ and $K_{s}$ are respectively the singular and nonsingular parts of the kernel in $\left(3.4 \mathrm{a}^{\prime}\right)$, the $f_{j}$ are defined in (3.6) and $w(t)$ is given by:

$$
w(t)=\frac{1}{h}\left[u\left(\tau_{+}+\frac{h}{2}, t\right)-u\left(\tau_{+}-\frac{h}{2}, t\right)\right] .
$$

The remaining integral was done analytically.

For all cases described below the grid ranged between -12 and 12 and contained 171 points. The time step is .025 , well within the stable region in all cases. Initial conditions were generated in the following way: expansions in the tail, (3.3), were input and smoothly connected (two continuous derivatives) by a combination of polynomial and exponential functions. The computations shown were performed on a VAX 11/780 at the University of Wisconsin at Madison, though others were done on the IBM 4341 of the Applied Mathematics Department at the California Institute of Technology.

Figure 1 shows the evolution, in a coordinate system moving with speed 4, of initial data which decays, at both $\pm \infty$, at a rate compatible with a wave of speed 4 .

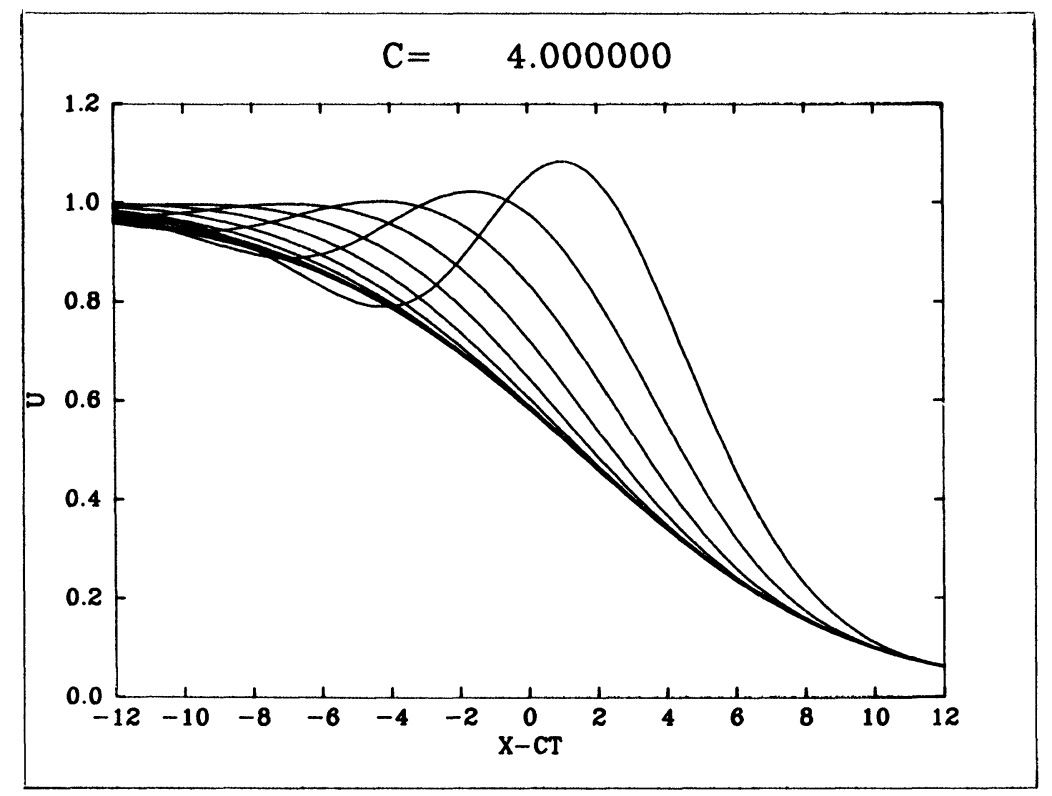

FIG. 1 
The initial data and solutions at intervals of 25 time steps are displayed. A steady state is reached which must be moving with speed 4 . Figure 2 contains the final state (solid line) of Fig. 1. This is the solution at $t=6.875$. Plotted with it is the traveling wave solution of speed 4 . This solution was found by a second order centered finite difference solution of the relevant steady problem on the same mesh as used in the time dependent case. The solution was required to agree with the time dependent one at the right-hand boundary while the condition:

$$
u_{x}=\bar{\kappa}_{+}(0 ; 4)(u-1)
$$

was used at $\tau_{-}$. (Note that $\bar{\kappa}_{+}(s ; c)$ is defined in (2.16).) The agreement between the two solutions is seen to be excellent.

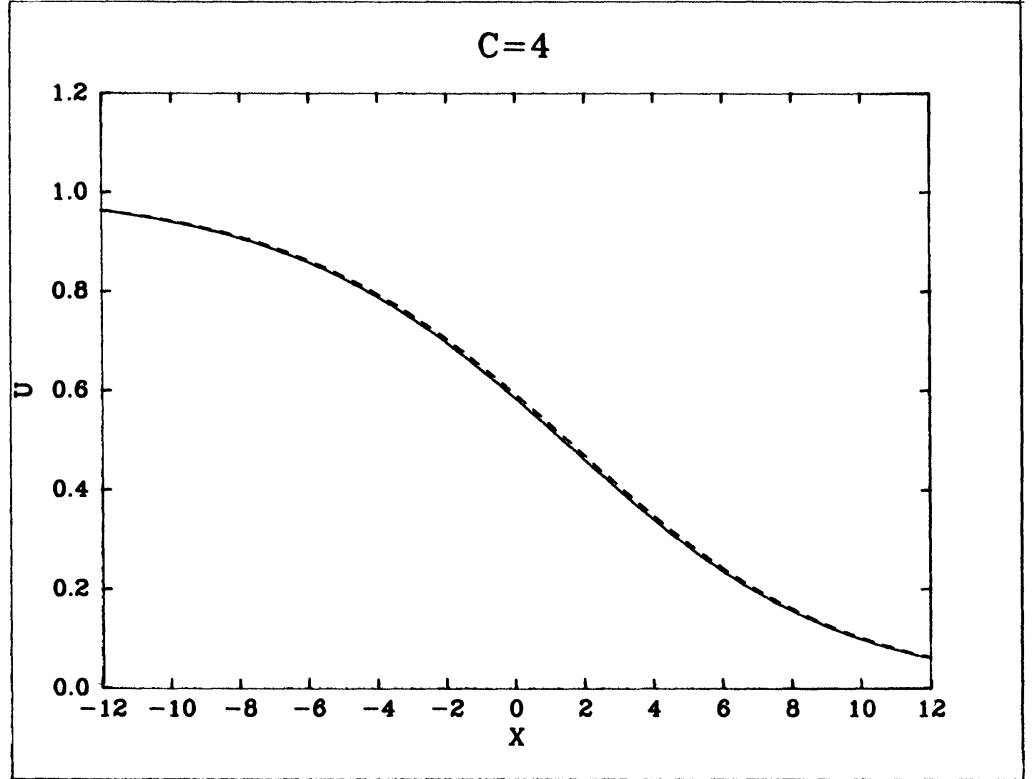

FIG. 2

We note that the boundary condition,

$$
u\left(\tau_{+}, t\right)=\text { constant, }
$$

leads to good results when the speed of the coordinate system is the same as the speed of the final state. For a more complicated problem, however, this might not be known in advance. Indeed, it might be the goal of the computation to determine it. As shown in Fig. 3, our conditions avoid this difficulty. This is the computed evolution in a coordinate system moving with speed 3 of the same initial data used to generate Fig. 1. The wave is seen to move to the right and, in fact, moves with relative speed 1 . This is confirmed in Fig. 4, a comparison of the solution at $t=6.875$ (solid line) and the wave of speed 4 of Fig. 2 translated to the right a distance of 6.875 . We believe the small error at the right boundary is due to the use of linearized boundary conditions.

Figure 5 displays the computed evolution, in a coordinate system moving with speed 4, of initial data with two decay rates in the right tail: one compatible with a wave of speed 4 , the other compatible with a wave of speed 3 . Here, the speed 4 part decayed at the large rate while the speed 3 part decayed at the slow rate. As predicted by the theory, a speed 3 wave is eventually reached. 


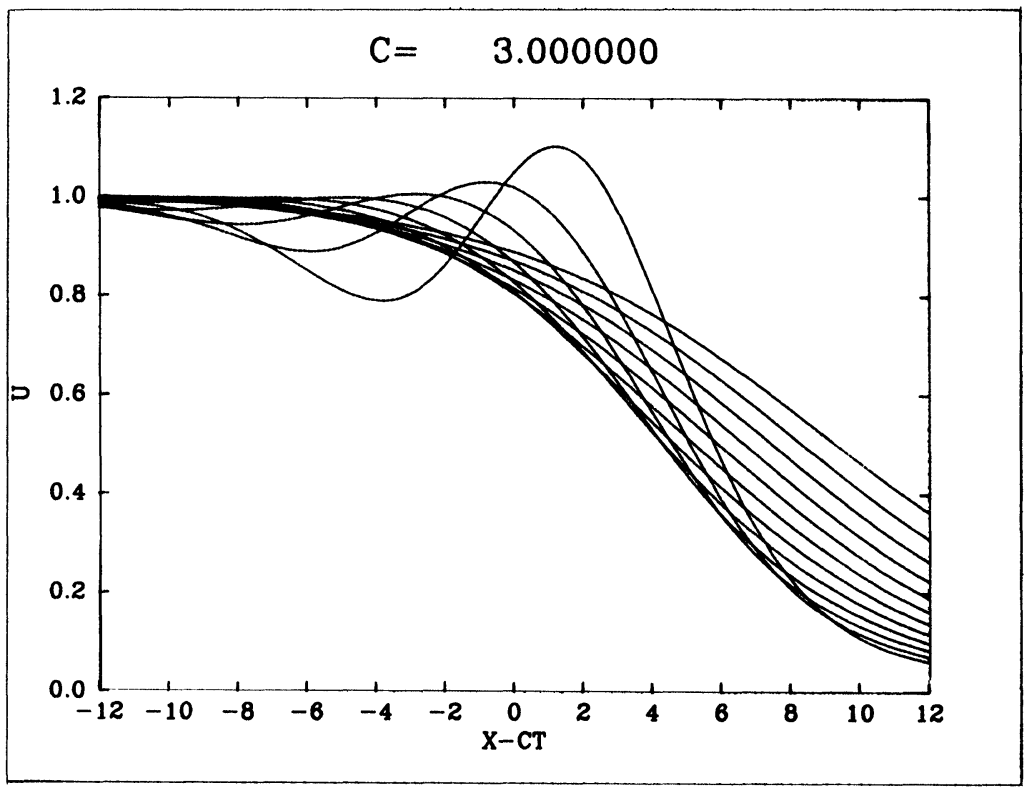

FIG. 3

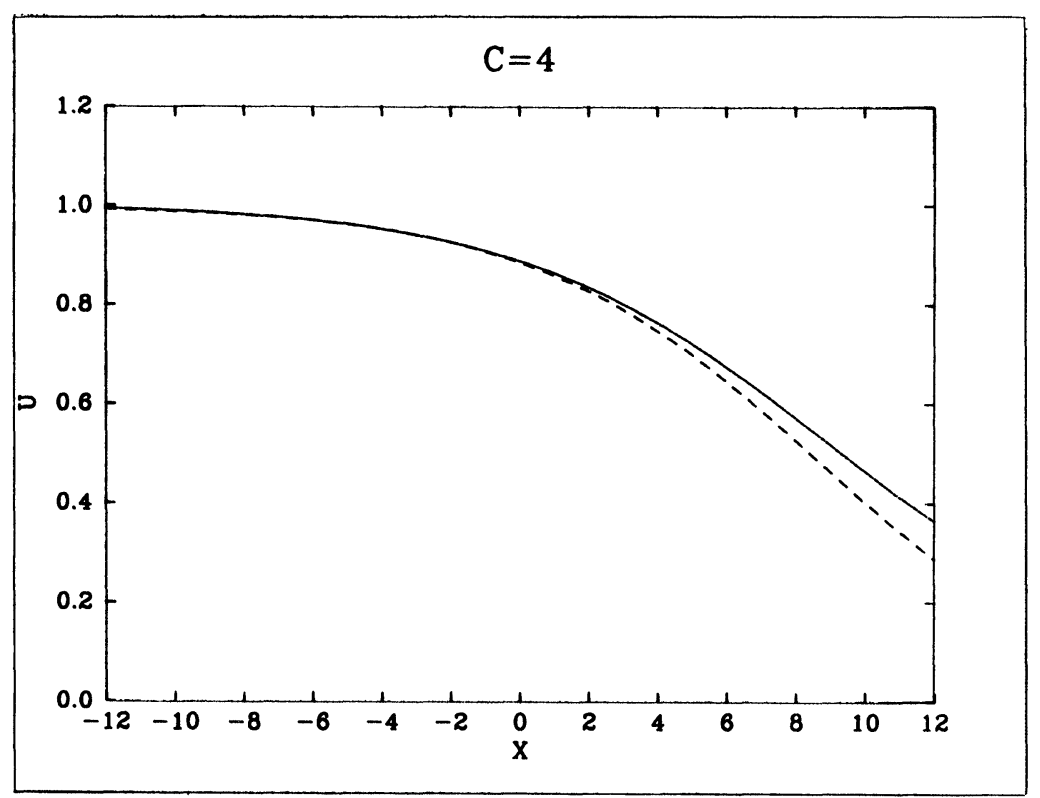

FIG. 4

We note that, as it is the initial data in the right tail which determines the wavespeed, it is the right-hand boundary condition which is important. Various choices for the left-hand boundary condition, for example $u=$ constant and $u_{x}=0$, were tried and led to good results.

In summary, we have shown that our boundary conditions consistently lead to correct long-time results while other simpler conditions do not. We believe that their 


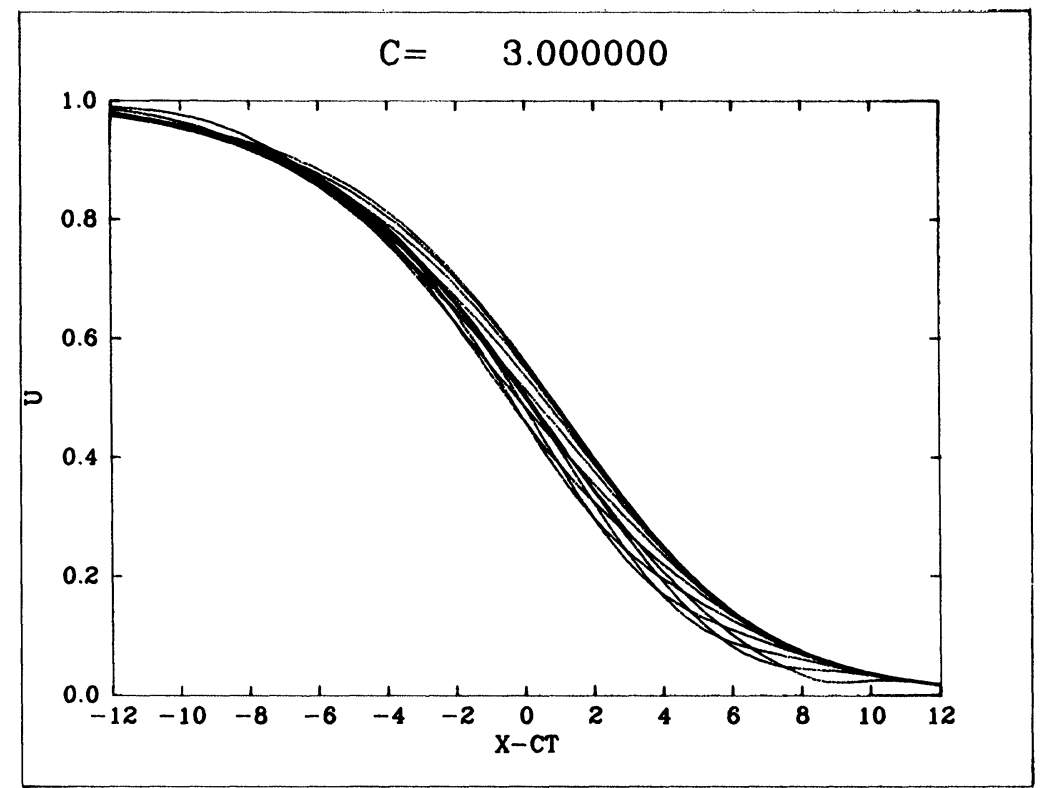

FIG. 5

generalization to more complicated problems, where the final state is not known a priori, will also give reliable results. It should be noted, however, that this has not yet been proved even in the simple case described here.

Acknowledgment. The authors thank Prof. J. D. Murray for bringing this problem to our attention.

\section{REFERENCES}

[1] J. Gazdag And J. Canosa, Numerical solution of Fisher's equation, J. Appl. Probl., 11 (1974), pp. 445-457.

[2] B. GUSTAFSSON AND H.-O. KREISS, Boundary conditions for time dependent problems with an artificial boundary, J. Comput. Phys., 30 (1979), pp. 333-351.

[3] P. HAGAN, Traveling wave and multiple traveling wave solutions of parabolic equations, SIAM J. Math. Anal., 5 (1982), pp. 717-738.

[4] - The instability of non-monotonic wave solutions of parabolic equations, Stud. Appl. Math. (1981), pp. $57-88$.

[5] T. HAGSTROM, Reduction of unbounded domains to bounded domains for partial differential equation problems, Ph.D. thesis, California Institute of Technology, Pasadena, 1983.

[6] T. HAGSTROM AND H. B. KELlER, Exact boundary conditions at an artificial boundary for partial differential equations in cylinders, SIAM J. Math. Anal., to appear.

[7] D. A. LARSON, Transient bounds and time-asymptotic behavior of solutions to nonlinear equations of Fisher type, SIAM J. Appl. Math., 34 (1978), pp. 93-103.

[8] P. MORAN, The Statistical Processes of Evolutionary Theory, Clarendon Press, Oxford, 1962, pp. $178-186$.

[9] F. Oberhettinger AND L. BADII, Tables of Laplace Transforms, Springer-Verlag, New York, 1973. 